\title{
Design of Ground Penetrating Radar Antenna for Detecting Soil Contamination at L-band Frequencies
}

\author{
Ahmad H. Abdelgwad, Tarek M. Said \\ Department of Electrical Engineering, Faculty of Engineering, Fayoum University, Egypt \\ aha05@fayoum.edu.eg,tms02@fayoum.edu.eg
}

\begin{abstract}
Maintenance of oil pipelines is an issue of great concern for oil companies. Soil contamination is caused by oil leaks from underground pipelines. Ground penetrating radar (GPR) is a rapid and relatively inexpensive technique used for locating and characterizing soil contaminated sites without producing fractures and causing further migration of contaminants. One of the most critical hardware components for the performance of GPR is the antenna system. The present paper reports on the design and simulation of a pyramidal horn antenna operating at L-band frequencies (1- $2 \mathrm{GHz}$ ) to detect soil contamination. A prototype model of the GPR system setup is developed to simulate the electromagnetic fields in different soil types. The dielectric permittivity of soil, needed in order to carry out simulations during the design process, is measured and analytically represented by Debye relaxation model. The contrast in the dielectric permittivity between contaminated and uncontaminated soils is the most important parameter to be considered for detecting the presence of contamination. The application of GPR is proved to be well-versed in the investigation of soil contamination.
\end{abstract}

Index Terms - soil contamination detection, ground penetrating radar, permittivity measurement, pyramidal horn antenna design

\section{INTRODUCTION}

Soil contamination is caused by underground pipeline rusting or leaking. Leaks and spills from the pipelines are a potential concern. If the pipelines are not appropriately maintained leaks may occur to the surrounding environment which, in turn, could contaminate the surrounding soil. The major causes of leaking of underground piping systems are cracking, drilling, and corrosion [1]. Contamination may spread over large areas and sometimes it is hard to locate. Different techniques are developed for detecting oil leakage from underground pipelines [2]. Leak detection using invasive techniques require drilling, soil sampling, and laboratory testing. These methods can promote further spread of contaminants in polluted areas. On the contrary, non-invasive techniques are relatively simple, inexpensive, and provide a rapid characterization of contamination without creating cracks.

Ground Penetrating Radar (GPR) is a non-destructive tool for non-invasive investigation [3]. GPR is a geophysical technique that can be used to investigate and map the dielectric properties of the subsurface [4]-[5]. It is based on measuring the electromagnetic pulses that being transmitted into the 
medium and then being collected by the receiver. Both of the transmitter and receiver are being placed in the antenna [6]. The success of GPR in detecting soil contamination depends on the contrast of electrical properties of both the clean and contaminated soil [7]. Consequently, direct measurement of the dielectric properties of the contaminated zone is effective in the assessment of detecting soil contamination [8]-[9].

Ground-penetrating radar antennas are usually located either near to or in contact with the soil surface. Therefore, the performance of GPR antenna system, such as operational frequency, transmitted power and antenna beamwidth, is directly depending on soil properties. The optimal antenna design must provide steady performance for different soil types and weather conditions. Dry soils are favorable for the GPR application, since higher radio-frequencies can be used for a given depth of investigation [10]. The capability of radar antenna in detecting the contaminant depends on both the required depth of penetration and the frequency of the antenna used [11]. The operating frequency of the GPR system is a trade-off. Low frequencies give better penetration yet low resolution so that soil contamination may not be detected. Contamination may be better detected utilizing higher frequencies yet the depth of penetration may be limited to only a couple of centimeters particularly in moist soil.

The present paper is organized as follows. In "Prototype model and dielectric modeling" section, a prototype soil model is introduced to investigate the effectiveness of GPR technology in detecting soil contamination. The situation incorporates a plastic tube installed in soil and filled with lubricant oil. Two scenarios are studied, the first is where the soil beneath the pipe is dry sand, and the second one is where the soil beneath the pipe is contaminated with oil due to pipe leaks. On the basis of such a description, two identical wideband pyramidal horn antennas that operate at L-band frequencies are then designed in "GPR antenna design" section to exhibit that microwave sensing is precisely able to discriminate between contaminated and clean soils. "Conclusions" section summarizes our work.

\section{PROTOTYPE MODEL AND DIELECTRIC MODELING}

The primary objective of this work is to research the potential of using GPR technology in detecting soil contamination caused by leakage that may occur in oil pipelines. To achieve this objective, a prototype model is precisely designed and simulated. The prototype model is essentially consist of a wooden box loaded with sand where a plastic pipe contains oil is incorporated in soil medium. Figure 1-(a) illustrates the geometry of the designed model and the optimized dimensions, searched for best performance, are listed in Table I. The prototype model for the situation outlined in Fig. 1 needs a dielectric modeling of mediums of interest. Propagation of microwaves in the soil medium is dictated by its dielectric permittivity. Accurate detection of the pollutants in the soil medium can be obtained by effectively measuring its dielectric permittivity [12]. 


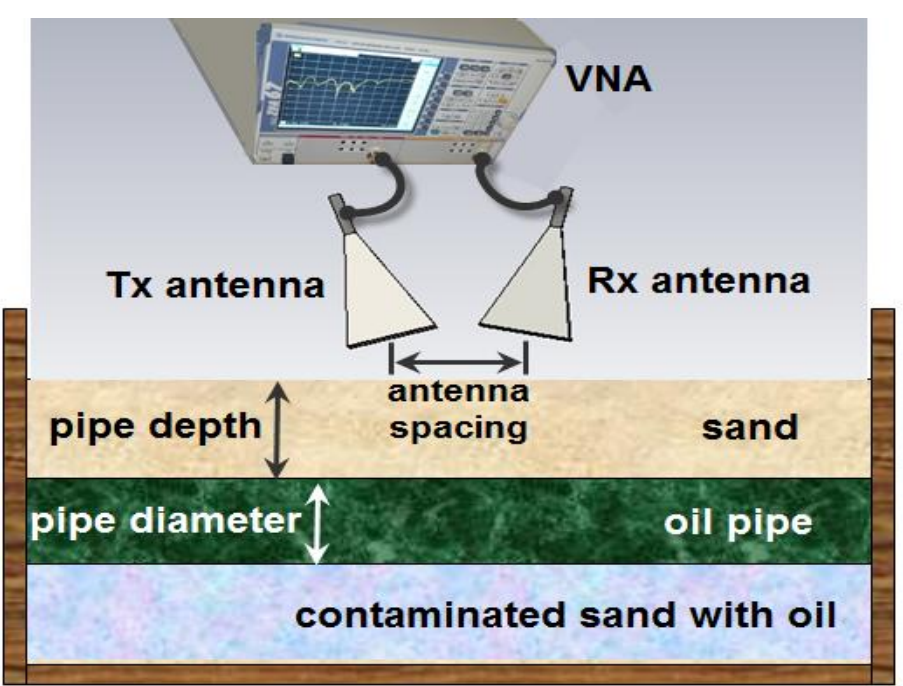

(a)

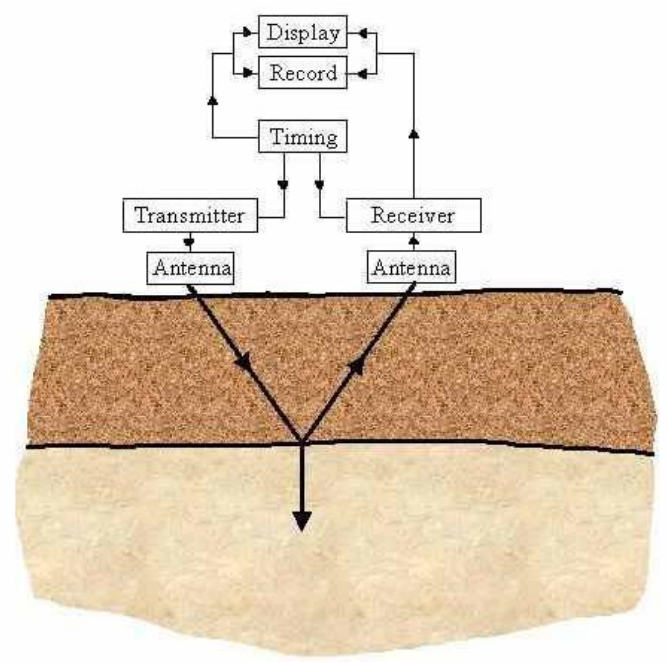

(b)

Fig.1 Soil contamination detection prototype model

TABLE I. OPTIMIZED DIMENSIONS OF MODEL

\begin{tabular}{cc}
\hline Parameter & value (cm) \\
\hline wooden box length & 150 \\
wooden box width & 100 \\
wooden box height & 100 \\
antenna height from ground surface (H) & 10 \\
antenna spacing (S) & 50 \\
pipe burial depth (D) & 40 \\
pipe diameter (d) & 10 \\
antenna tilting from vertical & $22^{\mathbf{o}}$ \\
\hline
\end{tabular}

The GPR used is homodyne where the detection is performed by extracting information encoded as modulation of the phase and/or frequency of the oscillating signal, by comparing that signal with a standard oscillation that would be identical to the signal if it carried null information. The utilized frequency modulated homodyne detection signifies a single frequency, in contrast to the dual frequencies employed in heterodyne detection. The radar pulses are used to image the subsurface. This nondestructive method uses electromagnetic radiation in the L- band of the radio spectrum, and detects the reflected signals from subsurface structures as shown in Fig. 1-(b).

Different techniques can be used for the measurement of the dielectric permittivity of materials in the microwave range. These techniques include transmission line systems (free-space, coaxial and waveguide), cavity and impedance techniques [13]. To measure the complex dielectric permittivity of soil samples, we have used the Dielectric Assessment Kit (DAK). DAK system performs simple, precise, and convenient dielectric measurements in the microwave frequency range. The experimental setup of DAK measurement system is shown in Fig. 2. DAK setup consists of a Vector Network Analyzer (VNA), open ended coaxial probe, and computer Software. The DAK probe has an impedance of $50 \Omega$ and it should be calibrated with respect to a consistent short circuit fitted at the 
probe face and a reference sample of known permittivity. The end of a coaxial line probe is then placed in contact with the soil sample and connected to the VNA to measure the complex reflection coefficient at the probe end. The soil medium surrounding the probe tip should be smooth and homogenous in order to eliminate phase distortions due to cable movements. The measured reflection coefficient is then converted to the complex permittivity of the sample under the test using DAK Software. The sample volume should be large enough and homogeneous enough to avoid any reflections from the sample's boundary which could influence the measurements.

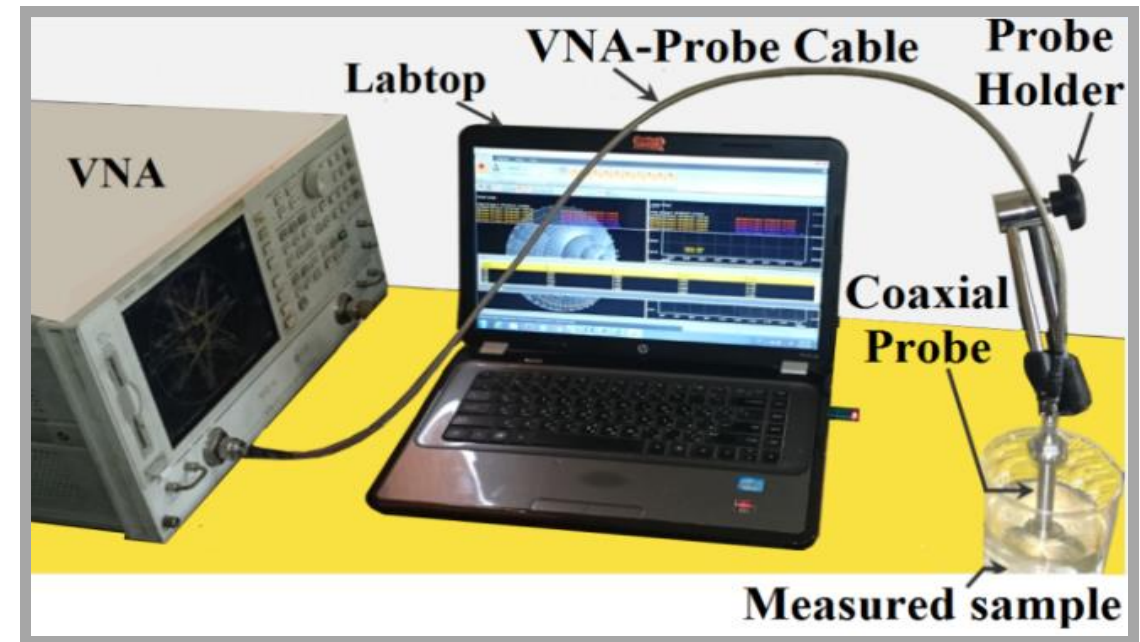

Fig. 2. DAK measurement system setup

Using the DAK system at room temperature, the complex dielectric permittivity of both clean and contaminated sandy soil samples is measured as a function of frequency. The measured complex permittivity of soil samples is then fitted to the Debye relaxation model [14]-[15]:

$$
\varepsilon=\varepsilon_{\infty}+\frac{\varepsilon_{s}-\varepsilon_{\infty}}{1-i f / f_{0}}+i a^{\prime \prime}
$$

where $\varepsilon_{\mathrm{s}}$ is the static permittivity, $\varepsilon_{\infty}$ is an extrapolated high frequency permittivity, $f_{o}$ is the relaxation frequency, $i$ is the complex number symbol $i=V_{-} 1$, and $a^{\prime \prime}$ is an estimated imaginary part representing the sand losses. A curve fitting routine is implemented using MATLAB software to search for the most appropriate values of fitting Debye relaxation parameters. The searching algorithm is based on the least square method. The objective of data fitting is to determine the values of the Debye relaxation model that almost match the measured data. The best Debye fitting parameters are listed in Table II for all measured soil samples. Relaxation models have been widely used by researchers to extrapolate the measured permittivity to higher frequencies. With an appropriate choice of model parameter, relaxation model could be used to predict the dielectric properties over the desired frequency range. 
TABLE II. DEBYE PARAMETERS OF TWO DIFFERENT SANDY SOILS

\begin{tabular}{ccc}
\hline Soil Type & Uncontaminated sandy soil & Contaminated sandy soil \\
\hline$\varepsilon_{\mathrm{s}}$ & 2.51 & 5.68 \\
$\varepsilon_{\infty}$ & 2.82 & 5.99 \\
$f_{\mathrm{o}}(\mathrm{GHz})$ & 0.249 & 0.412 \\
$a^{\prime \prime}$ & 0.003 & 0.285 \\
\hline
\end{tabular}

At microwave frequencies the value of the dielectric permittivity of a natural dry soil sample is related to its physical component. As a consequence of adding lubricant oil to the sandy soil sample, oil will infiltrate deeply into soil replacing air in pore spaces of the soil sample. The soil characteristics become more similar to characteristics of the lubricant oil hence its chemical and physical properties will alter causing the adjustments in its dielectric permittivity. The experimentally measured values and fitted data of the dielectric constant and the dielectric loss are shown in Fig. 3 for both clean and oil soil contaminated samples. The measurements indicate a noticeable increment in both dielectric constant and dielectric loss factor due to the existence of the lubricant oil. This contrast in dielectric properties will in turn reflect on the Debye model parameters.

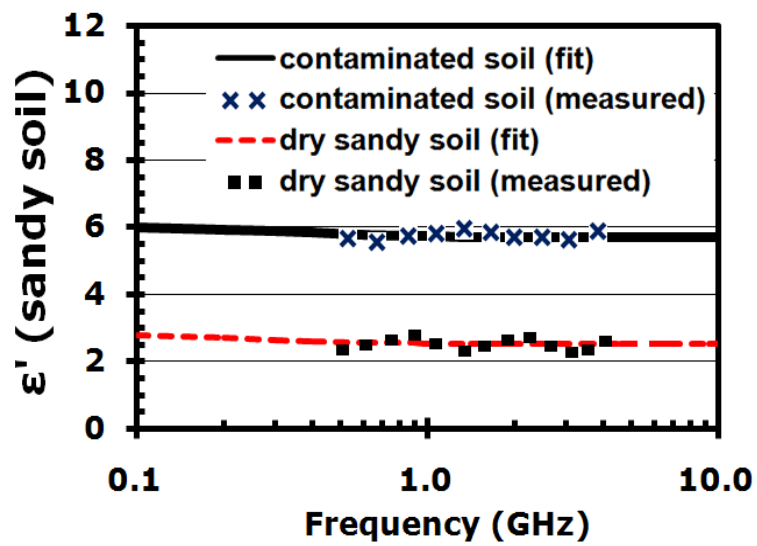

(a)

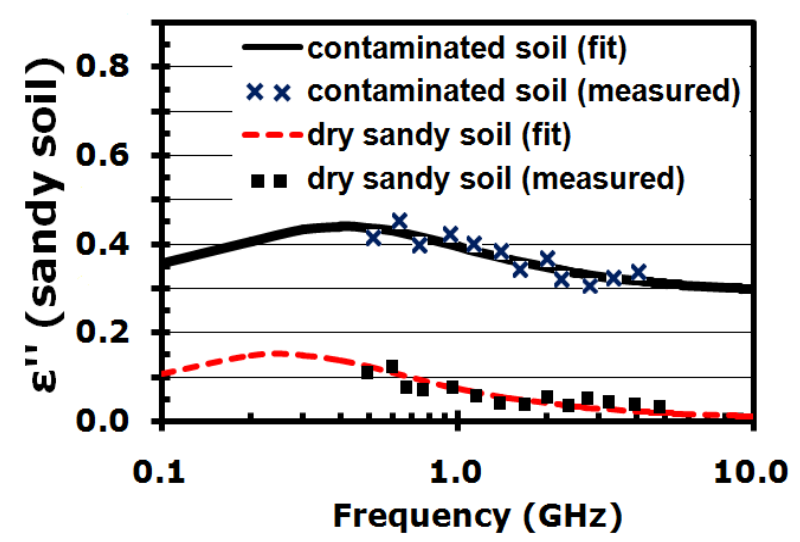

(b)

Fig. 3. Measured and curve-fitted permittivity of sandy soil versus frequency before and after contamination (a) dielectric constant (b) dielectric loss factor

Clearly, the amount of change in the dielectric properties of contaminated soil samples looks to be significant. In this way, ground penetrating radar (GPR) could be able to sense the contrast between reflected signals of both clean and contaminated soils. GPR equipment requires sufficient signal penetration. The penetration depth $\delta$ is the reciprocal of the absorption coefficient $\alpha$ of the medium ( $\alpha$ $=2 k_{0} n^{\prime \prime}$, where $k_{0}$ is the vacuum wave number and $n^{\prime \prime}$ is the imaginary part of the medium refractive index $n=\sqrt{ } \varepsilon$ ). Using equation (1), $\delta$ is computed and plotted over the L-band frequencies as shown in Fig. 4. We can notice that due to the increase of dielectric loss in the contaminated soil samples, the penetration depth will significantly decrease compared with its value in the clean soil samples. 


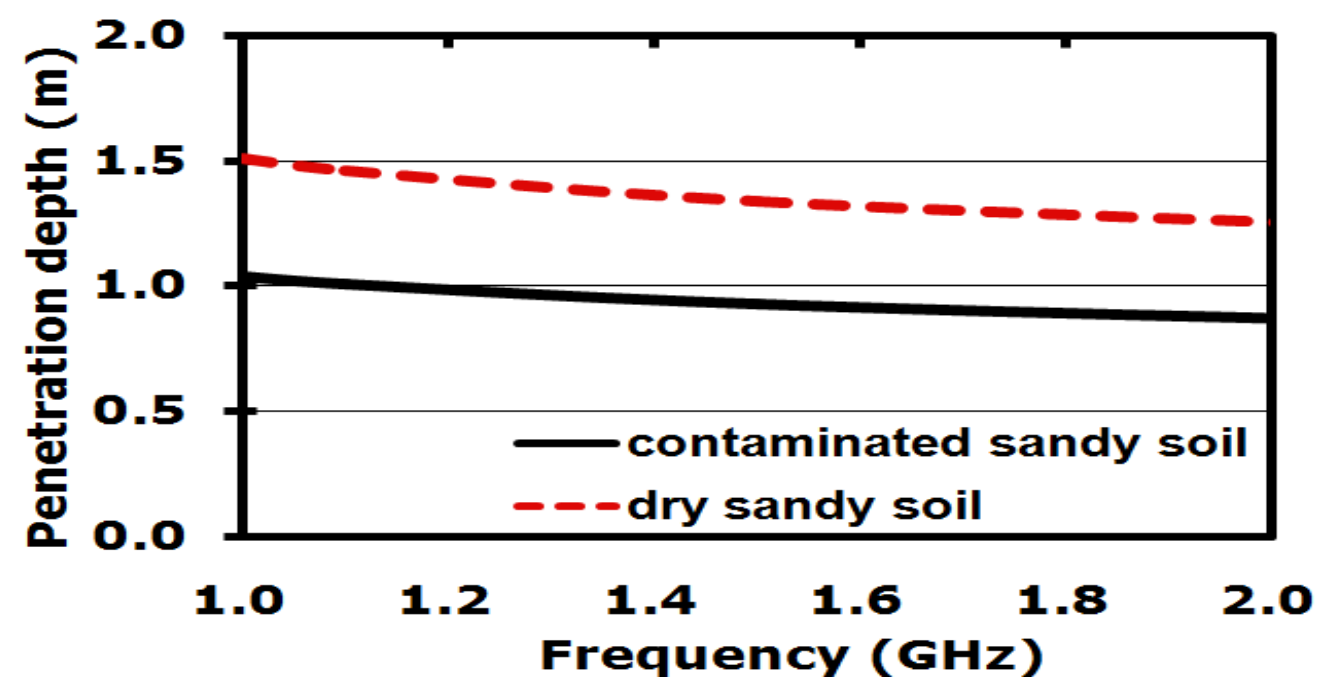

Fig. 4. Penetration depth of clean and contaminated sandy soils

\section{GPR HORN ANTENNA DESIGN}

The effective element of any GPR system is the antenna. In order to obtain deep penetration and fine resolution, the GPR antenna should have certain features like high directivity and efficiency, wide bandwidth, and proper impedance matching [16]. The horn antenna is the most useful form of antenna for use with microwave applications because of their advantages. Horn antennas are simple, inexpensive to be manufactured, and they are very easy to interface to waveguide. They also have excellent gain and directivity hence they are the best choice for many GPR applications. Horn antenna is applied in the proposed GPR model due to its good directivity, high gain, low side and back lobes, simple to feed, and high power-handling capabilities. The rectangular horns are preferably suitable for rectangular waveguide feeders. The horn facilities a gradual transition of the electromagnetic wave from the waveguide to the free-space.

For GPR antennas, the higher the frequency the less the signal is able to penetrate into the medium. However, higher frequency will provide better image resolution. Selecting the optimum GPR frequency is always a trade-off. Here, L-band frequencies are the most appropriate band to obtain the optimum results for the proposed model. A pyramidal horn antenna operating at L-band is designed using Computer Simulation Technology (CST) software program for the soil contamination detection model. The material used for designing the pyramidal horn and the waveguide is assumed to be perfect electric conductor (PEC) with thickness of $2 \mathrm{~mm}$. This guarantees that the microwaves transmitted in the waveguide is appropriately reflected and surface current on the waveguide does not produce much Ohmic loss. 


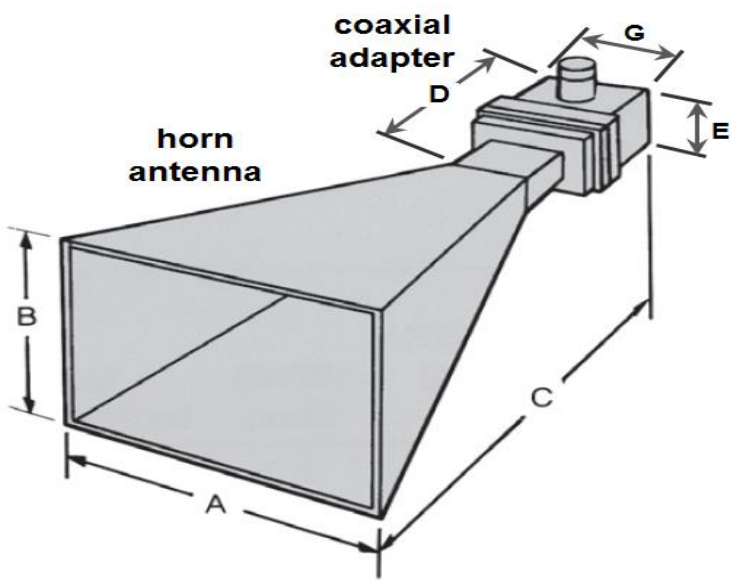

TABLE III. OPTIMIZED DIMENSIONS OF HORN ANTENNA

\begin{tabular}{cc}
\hline Antenna dimension & Unit (cm) \\
\hline Aperture width, $A$ & 41 \\
Aperture height, $B$ & 30 \\
Antenna length, $C$ & 80 \\
Waveguide length, $D$ & 20 \\
Waveguide height, $E$ & 12 \\
Waveguide width, $G$ & 24 \\
\hline
\end{tabular}

Fig. 5. Geometry of the proposed GPR horn antenna

Figure 5 depicts the structure of the proposed pyramidal horn antenna. The antenna is associated with a coaxial adapter which provides match between the waveguide and a $50 \mathrm{ohm}$ coaxial to be connected to the vector network analyzer (VNA) where the power flow can be in either direction. The overall optimized dimensions of the designed antenna, obtained utilizing pyramidal horn antennas dimensions mathematical relationships in [17], are organized in Table III.

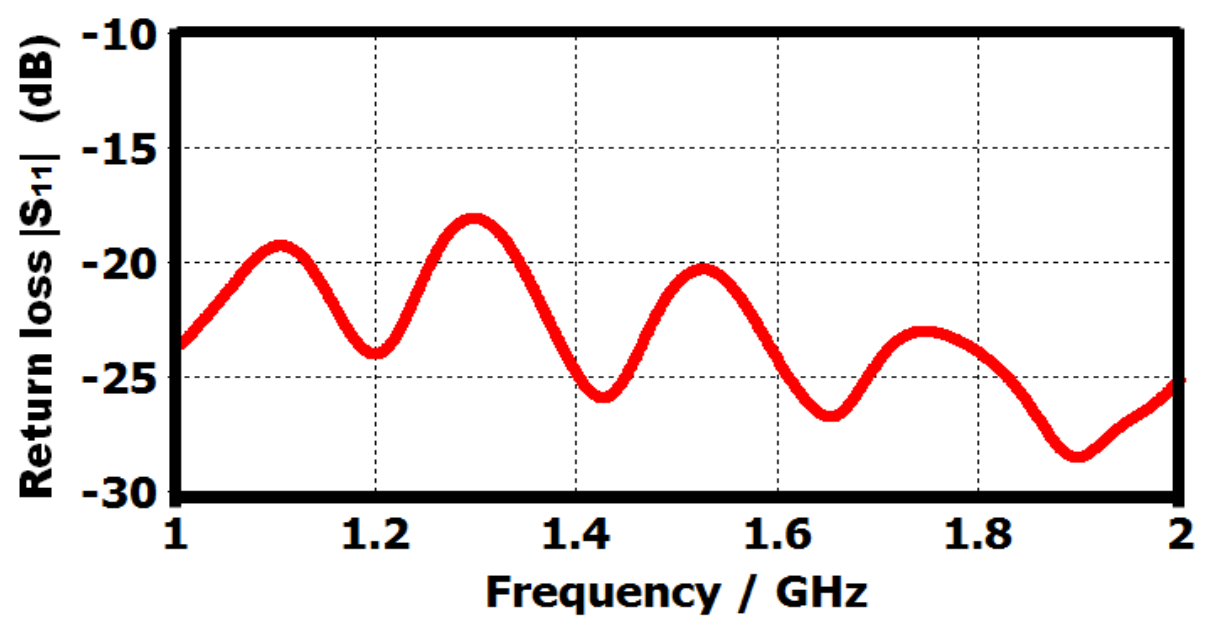

Fig. 6. Return loss of designed antenna at L-Band

Figure 6 represents the simulated return loss, $\left|S_{11}\right|$, of the designed GPR horn antenna. Figure 6 indicates that the designed horn antenna is operating at L-band frequencies with return loss lower than $-17 \mathrm{~dB}$. The obtained results confirm that most of the power will radiate in the free space. The polar farfield radiation pattern of the designed antenna is shown in Fig. 7 at 1, 1.5, and $2 \mathrm{GHz}$. The waveguide simulations indicate that the mode type is TE with wave impedance of $415.84 \mathrm{ohms}$. The cut off frequency is $0.635 \mathrm{GHz}$ and Beta is 28.48 [1/m]. Table IV indicates the gain, beamwidth and side lobe level over L-band frequencies. 
Farfield Gain Abs (Phi=90)
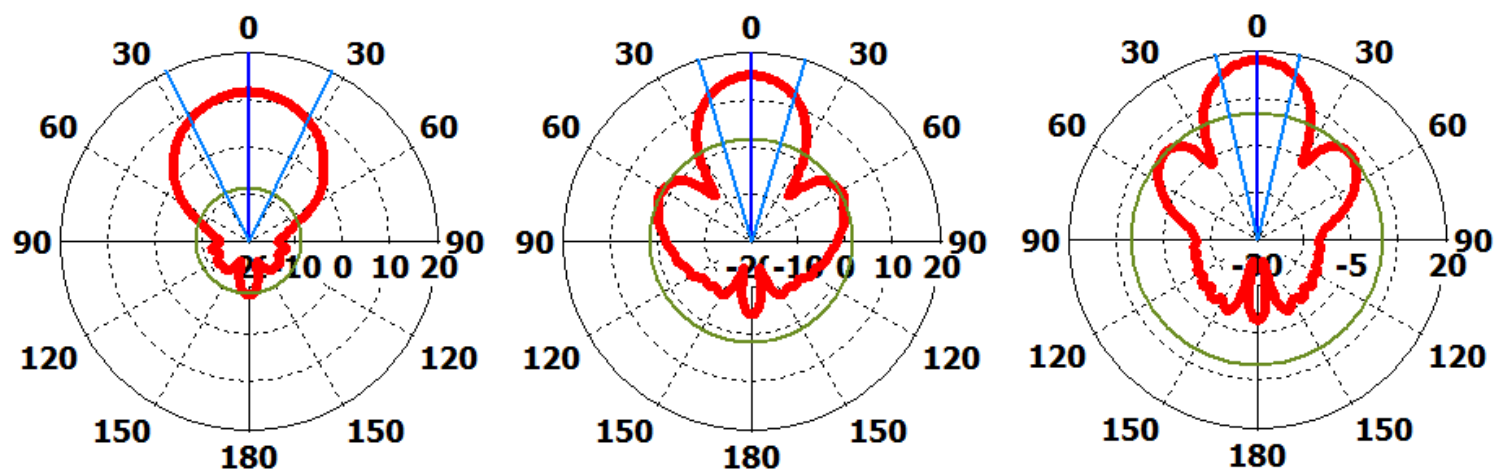

Theta / Degree vs. dB

Frequency $=1$
Main lobe magnitude $=11.4 \mathrm{~dB}$
Main lobe direction $=\quad 0.0 \mathrm{deg}$.
Angular width $(3 \mathrm{~dB})=52.3 \mathrm{deg}$.
Side lobe level $=-20.0 \mathrm{~dB}$

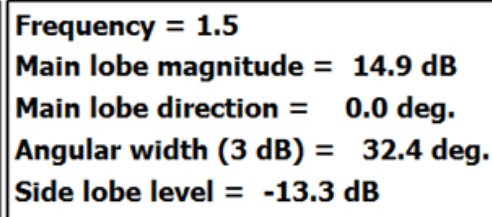

Fig. 7. Radiation pattern of the designed antenna at $1,1.5$ and $2 \mathrm{GHz}$

TABLE IV. FARFIELD RADIATION PARAMETERS OVER L-BAND

\begin{tabular}{|c|c|c|c|}
\hline $\begin{array}{c}\text { Frequency } \\
\text { (GHz) }\end{array}$ & $\begin{array}{c}\text { Main lobe } \\
\text { Gain } \\
\text { (dB) }\end{array}$ & $\begin{array}{c}\text { 3-dB } \\
\text { Beamwidth } \\
\text { (degrees) }\end{array}$ & $\begin{array}{c}\text { Side lobes } \\
\text { Level } \\
\text { (dB) }\end{array}$ \\
\hline 1.0 & 11.4 & 52.3 & -20.0 \\
\hline 1.1 & 12.2 & 47.4 & -22.0 \\
\hline 1.2 & 13.1 & 42.9 & -19.5 \\
\hline 1.3 & 13.8 & 36.4 & -13.3 \\
\hline 1.4 & 14.4 & 37.4 & -17.9 \\
\hline 1.5 & 14.9 & 32.4 & -13.3 \\
\hline 1.6 & 15.4 & 33.1 & -17.3 \\
\hline 1.7 & 15.9 & 29.1 & -12.8 \\
\hline 1.8 & 16.4 & 29.2 & -15.5 \\
\hline 1.9 & 16.7 & 26.9 & -12.8 \\
\hline 2.0 & 17.3 & 25.9 & -13.9 \\
\hline
\end{tabular}

Interestingly, the designed horn antenna provides directive radiation pattern with gain up to $11.4 \mathrm{~dB}$, $14.9 \mathrm{~dB}$, and $17.3 \mathrm{~dB}$ at $1 \mathrm{GHz}, 1.5 \mathrm{GHz}$, and $2 \mathrm{GHz}$ respectively; this high gain helps the microwave signals to penetrate the soil deeper beneath the ground surface. Furthermore, the very low side lobe level and the narrow beam width of the antenna diminish the coupling between the transmitting and receiving antennas. This in turn enhances the detection of soil contamination. 


\section{RESULTS AND DISCUSSIONS}

GPR system utilizes microwave signals to collect subsurface information by detecting the reflected signals of subsurface structures. The receiving antenna detects variations in the reflected return signal when the radiated wave strikes buried objects with different dielectric constants. The prototype landmine detection model illustrated in Fig. 1-(a) is realistically implemented using the CST Microwave Studio to ascertain the behaviour of the materials of interest. The antenna structures are modeled using $8.4 \times 10^{6}$ mesh-cells with a grid resolution of 40 steps per wavelength, which ensured the convergence properties of the fields. The simulation is performed with $-40 \mathrm{~dB}$ accuracy to provide very precise simulation results.

There are three GPR radar architectures which are mono-static, bi-static and multi-static architectures. In a mono-static architecture, a single antenna is used to transmit and to receive microwave signals. In bi-static architecture, two separated antennas are required for both transmission and reception. In multi-static radar system, the transmitting signal is accomplished by a single antenna, and multiple receiving antennas are used for the receiving of signals. In the current study, bistatic radar system is introduced. One of the most important issues that should be controlled in the bi-static radar architecture is the direct coupling between the transmitting and receiving antennas. In order to accomplish the best performance, the direct coupling ought to be minimized to increase the ability of the GPR to discriminate between the reflected signals of the buried object. The direct coupling could be controlled by the antennas spacing (S) and tilting (orientation). With the increase of antenna spacing, the direct coupling decreases. However, the received reflected signal of the buried object likewise becomes weaker and vice versa. The optimized antenna spacing is found to be $50 \mathrm{~cm}$ at which the direct coupling is minimized and in meanwhile the received reflected signals of the buried object is significantly notable. Figure 8 depicts the direct coupling between the transmitting and receiving antennas over L-band.

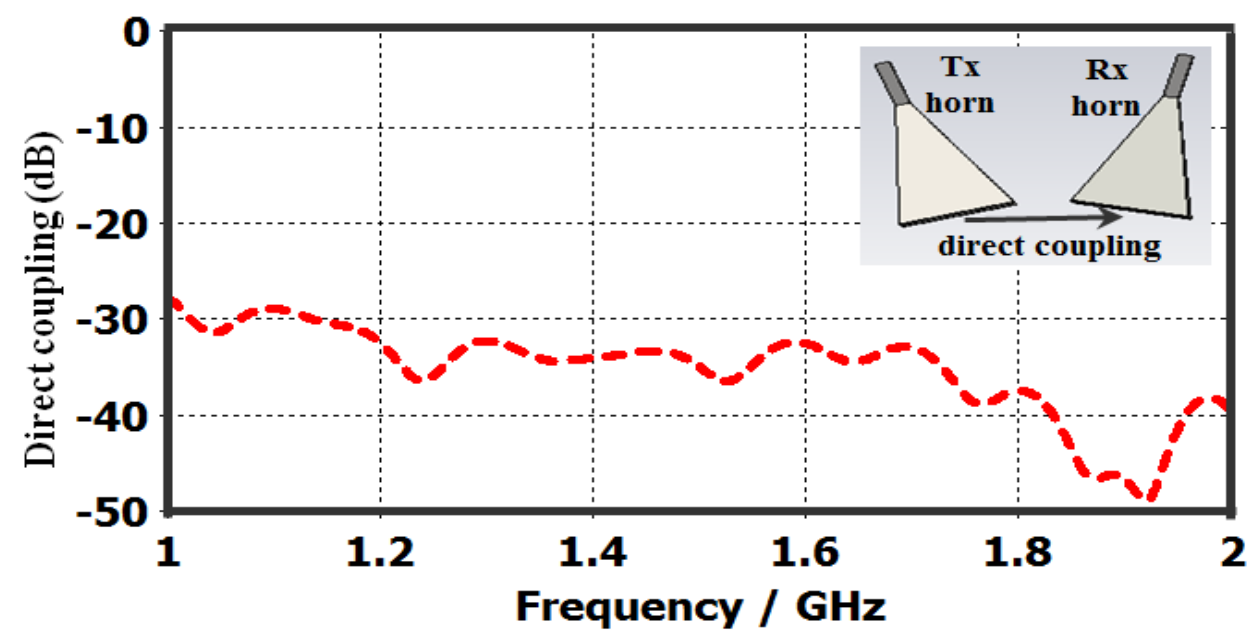

Fig. 8. Direct coupling between the transmitting and receiving antennas over L-band 
The capability of the GPR system to detect soil contamination can be measured by the change of the electromagnetic coupling, $\left|S_{21}\right|$, between the transmitting and receiving antennas due to the presence of contamination. The coupling coefficient, $\left|S_{21}\right|$, is simulated at L-band frequencies. A simulation setup is realistically implemented in the CST Microwave Studio. Two models are presented; the first one is where oil is placed inside the pipe as portrayed in Fig. 1-(a) and surrounded by dry sandy soil while the second one is where the layer of sand underneath the pipe is contaminated due to oil leak.

The coupling coefficient, $\left|S_{21}\right|$, for both models is compared in Fig. 9 with the dimensions introduced in Table I. We see that two resonance dips are lower than $-12 \mathrm{~dB}$ over entire frequency range, which implies that impedance matching bandwidth of the antenna is achieved only at these dips. The results show a significant increase in the coupling coefficient of contaminated soil model compared to uncontaminated soil model. It is additionally observed that for both models, the coupling coefficient decrease with the increase of frequency due to the increase of attenuation of soil with the increment of frequency. Also, three different cases are investigated: the increase of moisture content of soil, the depth at which the pipe is buried, and the change of spacing between the transmitter and receiver antennas.

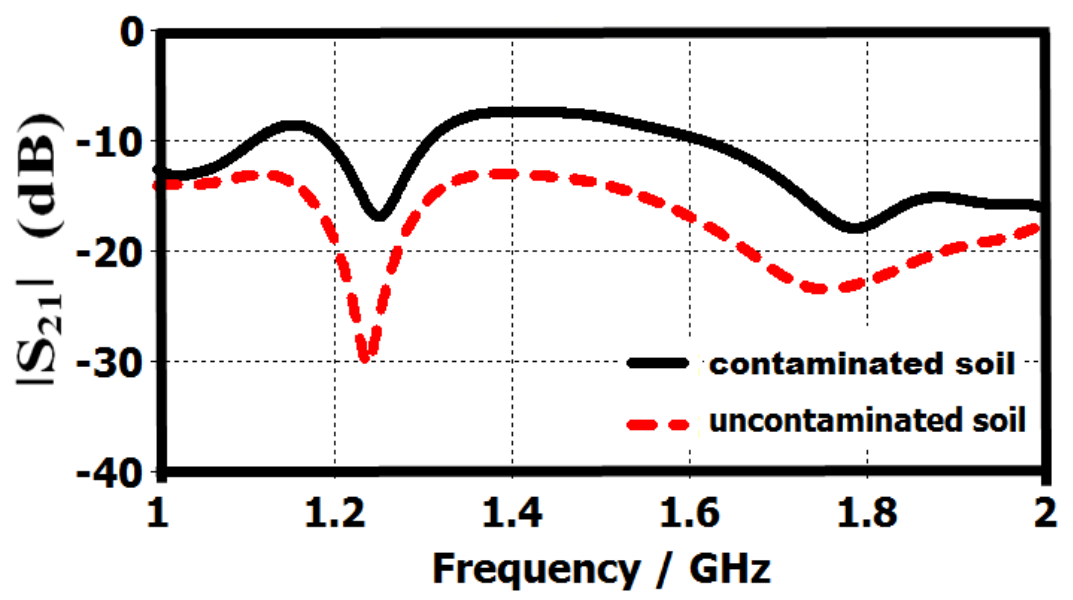

(a)

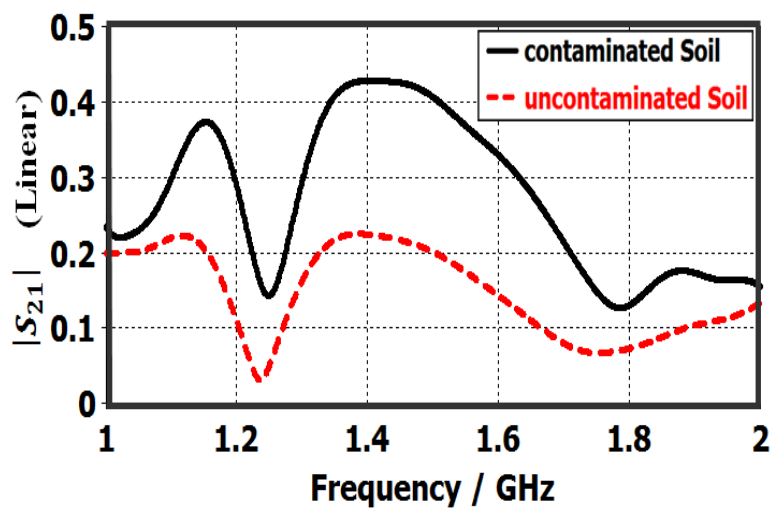

(b)

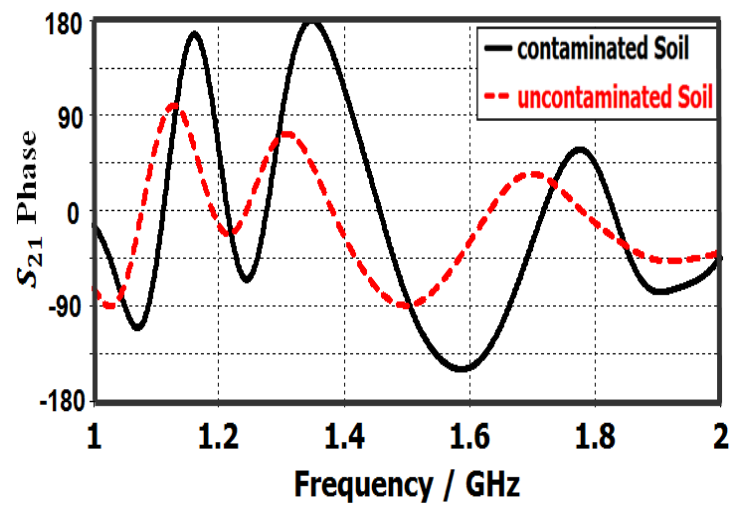

(c)

Fig. 9. Simulated coupling coefficient $S_{21}$ for contaminated and uncontaminated dry sandy soils (a) magnitude in $\mathrm{dB}$ (b) magnitude linear (c) phase 


\section{A. Effect of soil moisture}

The penetration depth of GPR system in soil medium being profiled depends on the operating frequency band as well as the soil conductivity. Applications which require deeper penetration in soil medium requires a lower frequency. Conversely, soil with high electrical conductivity will have lower penetration depth. Highly conductive soil will absorb the energy faster than a lower conductive soil. Moist soil is more conductive than dry sandy soil as it contains high water content, so penetration is only a couple of centimeters. As a result, dry sandy soils are considered to be highly suited to GPR antennas with low frequency than moist soils. It is anticipated, from the previous sub-section, that a significant contrast exists in the reflected signal of both contaminated and uncontaminated sandy soil. By replacing the dry sandy soil with moist sandy soil with dielectric constant of 13 and dielectric loss tangent of 0.29 [18], we notice that this contrast becomes insignificant as shown in Fig. 10. Presumably, It can be seen that $\left|S_{21}\right|$ of moist soil is more reduced than that of dry soil since moist soil has high electric conductivity which increases the attenuation. The results clarify, as expected, that oil contaminants are effectively detected in sandy soils, yet detection becomes infeasible in wet soils which is confirmed by all previous studies $[19,20]$.

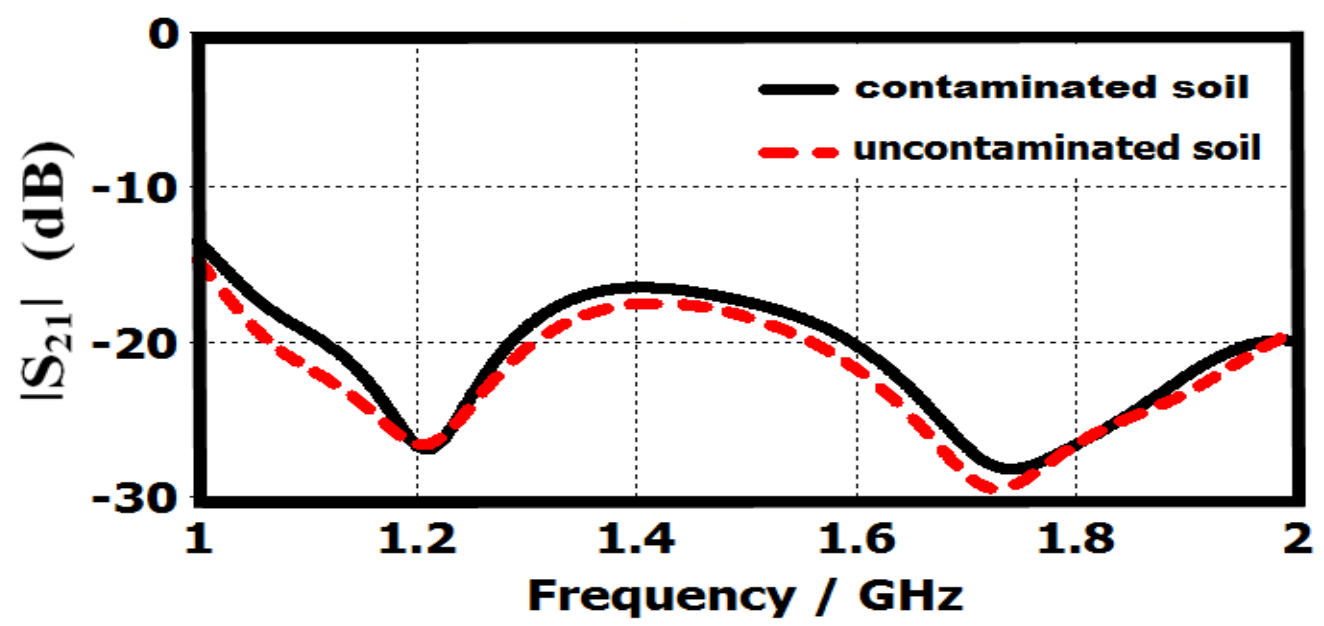

Fig. 10. Simulated $\left|S_{21}\right|$ coefficient for contaminated and uncontaminated moist sandy soils

\section{B. Effect of pipe burial depth}

Figure 11 demonstrates the consequence of increasing the depth (D) at which the pipe is buried from $40 \mathrm{~cm}$ to $50 \mathrm{~cm}$ on the coupling coefficient, $\left|S_{21}\right|$, while as all other dimensions organized in Table I are used. The results indicate that while the pipe burial depth increases, the coupling coefficient $\left|S_{21}\right|$ show a slightly decrease for both contaminated and uncontaminated soil. As the antenna to ground spacing increases, the antenna radiation pattern results in reduction of the received signal from the contaminated soil layer. This indicates that if the contaminated area is near to the surface of the ground, it becomes easier to be detected. The contaminated area most likely decreases the backscattered power by a considerable amount due to the increased attenuation. Hence, it is more challenging to detect deeper soil contamination. 


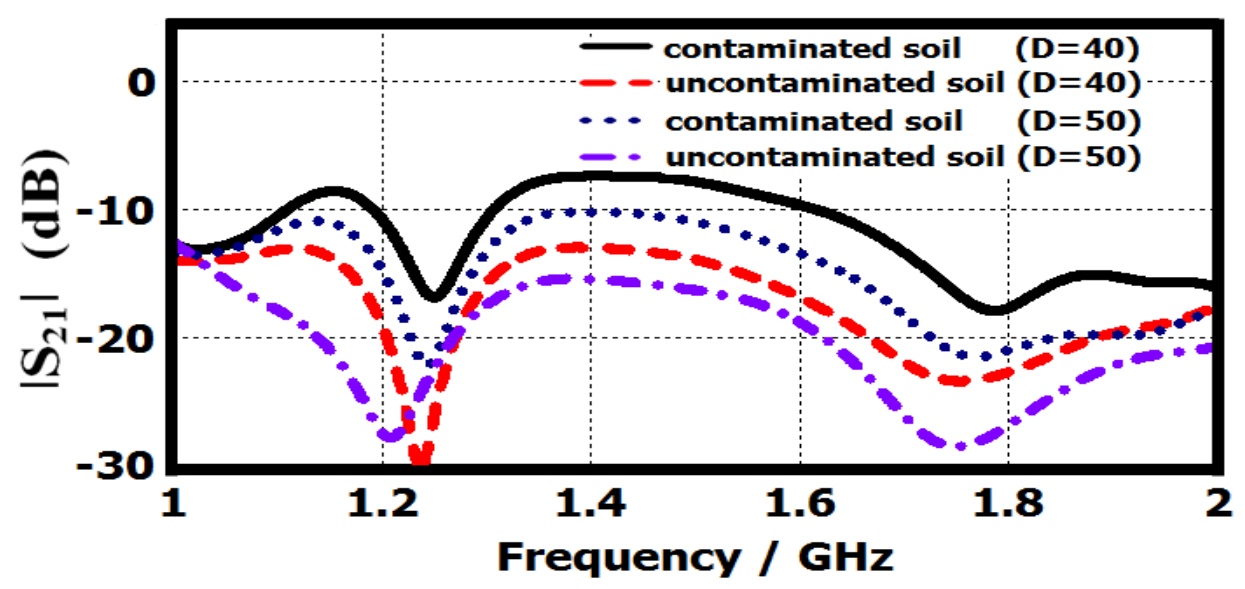

Fig. 11. Simulated $\left|S_{21}\right|$ coefficient for contaminated and uncontaminated dry sandy soils plotted for different pipe burial depth

\section{Effect of antenna spacing}

The determination of spacing between the transmitting and receiving antennas (S) and the tilting (orientation) of the antennas are very critical as they control the direct coupling between the transmitting and receiving antennas In order to accomplish the best soil contamination detection, this direct coupling ought to be minimized. With the increase of the spacing between the two antennas, the direct coupling decreases. However, the received reflected signal from the contaminated soil likewise becomes weaker. The optimized spacing between the transmitter and receiver antennas is $50 \mathrm{~cm}$ at which the direct coupling is minimized and meanwhile the received reflected signal from underground contaminated soil is significantly notable. Figure 12 illustrates the effect of increasing the direct coupling between the transmitting and receiving antennas by decreasing of antenna spacing (S) from $50 \mathrm{~cm}$ to $40 \mathrm{~cm}$ while keeping all other dimensions constant as in Table I. The comparison is presented over the entire range of frequency for the clean and contaminated soils as shown in the figure. The results indicate that as the antenna spacing increase, considerable increase occurs in $\left|\mathrm{S}_{21}\right|$ at the resonance dips of soil models due to the decrease of the direct coupling between the two antennas.

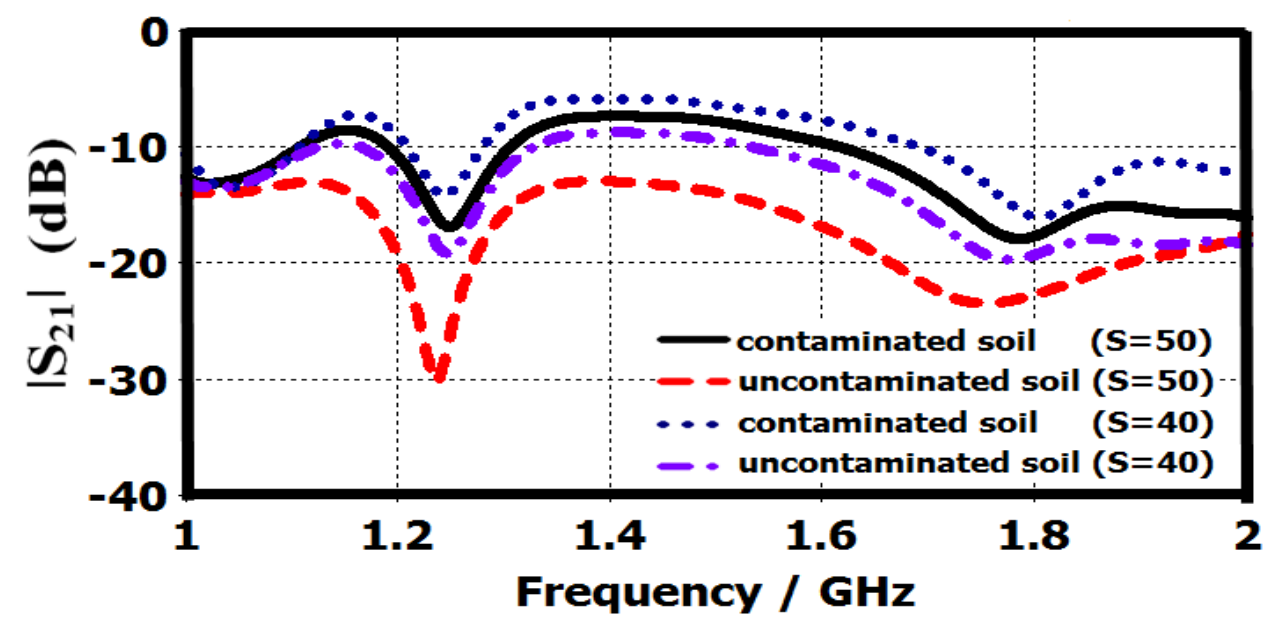

Fig. 12. Simulated $\left|S_{21}\right|$ coefficient for contaminated and uncontaminated dry sandy soils plotted for different antenna spacing 


\section{CONCLUSION}

The present study discuss the validity and effectiveness of utilizing GPR as a tool for detecting soil contamination due to leakage of oil from underground oil pipelines. Supported by full-wave electromagnetic simulations, we have discussed analytical aspects and design considerations of a prototype model of a pipe filled with lubricant oil and buried in sandy soil. The model is used to evaluate electromagnetic measurements taken in contaminated soil due to oil pipeline leaks. Two identical horn antennas operating at L-band frequencies has been designed and exploited for bi-static GPR system. Based on this design, it is proved that there is a noticeable contrast in the reflected signal in the presence and absence of oil leaks. One finding in this work is that the performance of the GPR system is highly dependent on three parameters: soil moisture content, pipe burial depth, and the antennas configuration. The obtained results indicate that GPR system can be customized for detecting soil contamination.

The work described in this paper has enormous potential for further research. Some of future possibilities resulting from the current research include:

(1) Fabrication of the designed pyramidal horn antenna and measurement of its parameters then comparing the measured values with simulated parameters in this study.

(2) Performing the real-world laboratory experiments of soil contamination detection model exhibited in the current study and compare the laboratory measurements with the simulated results presented in this work.

(3) Investigation of whether it is feasible to determine the proportion of soil contamination by utilizing samples contaminated with different concentrations and studying the impact on reflected signals to the ground penetrating radar receiving antenna.

(4) Investigation of this study utilizing multi-static radar architecture where multiple receiving antennas in an array configuration are used for the receiving of signals and compare the results with current study.

\section{REFERENCES}

[1] S. Yanxun, W. Yani, Q. Hui, and F. Yuan, "Analysis of the groundwater and soil pollution by oil leakage," Procedia Environmental Sciences, vol. 11, pp. 939-944, 2011

[2] M. Huang, C. Lui, L. C. Shen, and D. Shattuck, "Monitoring soil contamination using a contactless conductivity probe," Geophysical Prospecting, vol. 43, pp.759-778, 1995

[3] R. G. Pîrnăua, A. Mihu-Pintilieb, G. Bodib, A. Asănduleseic, and L. Niacşu, "Ground penetrating radar as noninvasive method used in soil science and archaeology," Soil Forming Factors and Processes from the Temperate Zone, vol. 13, no. 1 , pp. 15-31, 2014

[4] S. Cardimona, "Subsurface investigation using ground penetration radar," Proceeding of 2nd Annual Conference on the Application of Geophysical and NDT Methodologies to Transportation Facilities and Infrastructure, 2002

[5] A. H. Abdelgwad, T. M. Said, and A. M. Gody, "Developing of A Ground Penetrating Radar Antenna for Detecting Water Pollution in Underground Pipelines," International Journal of Microwaves Applications, vol. 4, no. 1, 2015

[6] H. M. Jol, "Ground penetrating radar: theory and applications," Elsevier, Oxford, UK.,2009

[7] A. Kaya and H. Fang, "Identification of contaminated soils by dielectric constant and electrical conductivity," J. Environ. Eng., vol. 123, no. 2, pp. 169-177, 1997

[8] S. Darayan, C. Liu, L. C. Shen and D. Shattuck, "Measurement of electrical properties of contaminated soil," Geophysical Prospecting, vol. 46, pp. 477-488, 1998 
[9] R. K. Rowe, J. Q. Shang, and Y. Xie, "Complex permittivity measurement system for detecting soil contamination," Canadian Geotechnical Journal, vol. 38, no.3, pp. 498-506, 2001

[10] N. Ahmadian, S. Hasan, and O. N. Calla, "Permittivity and backscattering coefficient of diesel oil contaminated soil at C band (5.3 GHz)," International Journal of Microwave Science and Technology, vol. 2013, pp. 1-9, 2013

[11] A. H. Abdelgwad and T. M. Said, "Measured dielectric permittivity of contaminated sandy soil at microwave frequency," Journal of Microwaves, Optoelectronics and Electromagnetic Applications, vol. 15, no. 2, 2016

[12] A. R. Von Hippel, "Dielectric Materials and Applications," MIT Press, Cambridge, Mass., UAS, 1961

[13] M. S. Venkatesh and G. S. V. Raghavan, "An overview of dielectric properties measuring techniques," Canadian Biosystems Engineering Journal, vol. 47, no. 7, pp. 15-30, 2005.

[14] R. L. Van Dam, B. Borchers, and J. M. H. Hendrickx, "Methods for prediction of soil dielectric properties: A review," In Detection and Remediation Technologies for Mines and Minelike Targets XProc. SPIE, vol. 5794, pp. 188-197, 2005

[15] C. Mätzler, "Thermal microwave radiation- Applications for remote sensing," IET Electromagnetic Waves Series 52, London, UK, 2006

[16] P. Cao, Y. Huang and J. Zhang, "A UWB monopole antenna for GPR applications," 6th European Conference on Antenna and Propagations EuCAP, 2012

[17] C. A. Balanis, "Antenna Theory: Analysis and Design,” John Wiley and Sons Inc., Hoboken, New Jersey, 2005

[18] H. Trang, "Simulation of mine detection over dry soil, snow, ice and water," Proc. SPIE, vol. 2765, pp. 430-440, 1996

[19] A. H. Abdelgwad, T. M. Said, A. M. Gody, "Microwave Detection of Water Pollution in Underground Pipelines," International Journal of Wireless and Microwave Technologies, IJWMT, vol.4, no.3, pp. 1-15, 2014

[20] A. H. Abdelgwad, T. M. Said and A. M. Gody, "A Non-destructive Electromagnetic-based Model for Detecting Water Pollution in Underground Pipelines," Al-Azhar University Engineering Journal, JAUES, Vol. 9, No. 3, pp.1-8, 2014 